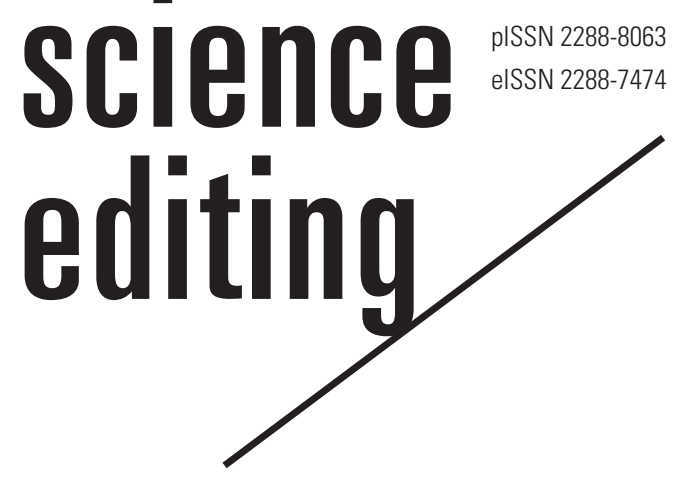

\title{
Bibliometric analysis of publications from North Korea indexed in the Web of Science Core Collection from 1988 to 2016
}

\author{
Geum Hee Jeong', Sun Huh² \\ 'Division of Nursing, Hallym University, Chuncheon; ${ }^{2}$ Department of Parasitology and Institute of Medical Education, College of \\ Medicine, Hallym University, Chuncheon, Korea
}

\section{Abstract}

The aim of this study was to analyze the bibliometric characteristics of publications from North Korea indexed in the Web of Science Core Collection from 1988 to 2016. We hypothesized that the main research area would be the physical sciences, and that the number of articles would continually increase over time. The Web of Science Core Collection was searched using the terms "North Korea" OR "Democratic People's Republic of Korea" OR "DPRK" in the address field of the basic search on February 2, 2017. The country of the co-authors, affiliations, journals, annual number of publications, and research fields were analyzed. Additionally, the articles by North Korean authors only were analyzed for the same parameters. A total of 318 articles from North Korea were found. The most frequent countries of collaboration were China, Germany, and Australia. Kim Il Sung University produced the most articles. The main research fields were physics, mathematics, and materials science. The categories of the journal titles corresponded to the research fields. The rapid increase in the number of articles in 2015 and 2016 was remarkable, although this increase started from a very small baseline number of publications. The results of the analysis of the 46 articles published by North Korean authors only were equivalent to the results for the 318 articles presented above. Our hypotheses were confirmed. The surge of articles in 2015 and 2016 may represent the recent efforts by the North Korean government to emphasize scientific research and development. It is anticipated that the productivity of North Korean researchers in terms of publications in international jour-

Received: February 7, 2017 Accepted: February 13, 2017

\section{Correspondence to Sun Huh} shuh@hallym.ac.kr

ORCID

Geum Hee Jeong

http://orcid.org/0000-0002-2254-1962 Sun Huh

http://orcid.org/0000-0002-8559-8640 nals will increase dramatically based on the above trends, although the publication baseline is very low.

Keywords

Bibliometrics; Democratic People’s Republic of Korea; Publications; Research 


\section{Introduction}

North Korea (the Democratic People's Republic of Korea) is one of the most closed nations in the field of journal article publications. Although some technological fields in North Korea are top-notch (for example, nuclear and military arms technology), few articles have been published in the international literature databases by North Korean researchers [1]. Most research results by North Korean researchers have been published in journals in North Korea. Scientific, technological, and medical (STM) journals from North Korea are listed in NK Scholar, which is available at http://www.nkscholar.com/. This site is the literature database of 26 STM journals from North Korea, containing 85,147 articles as of January 27, 2017. There are other STM, social sciences, arts, and humanities journals; however, it is difficult to find a comprehensive list of such journals. It is also difficult to read the journal articles published in North Korean journals. For those who can read Korean, articles can be purchased from NK Scholar. Since the user interface of the database is only in Korean, it is difficult for international researchers to access the information.

Some articles from North Korean researchers have been introduced to the world through international literature databases such as Web of Science, PubMed, and Scopus. The aim of this study was to analyze the bibliometric characteristics of 318 publications from North Korea indexed in the Web of Science Core Collection from 1988 to 2016. This study may be able to identify trends in research activities and collaboration with foreign researchers by researchers based in North Korea. We hypothesized that the main research area would be the physical sciences due to the absence of sufficient resources for the life sciences, and that the number of articles from North Korean authors would exhibit a continuous increase, based on previous bibliometric results [1].

\section{Methods}

The Web of Science Core Collection was searched using the terms "North Korea" OR "Democratic People's Republic of Korea" OR "DPRK" in the address field of the basic search on February 2, 2017. The following settings were used: Science Citation Index Expanded-1900 to present; Social Sciences Citation Index-1956 to present; and Arts \& Humanities Citation Index-1975 to present. This resulted in 401 hits. Only articles with affiliations with North Korean institutions were included in the analysis because some articles with affiliations in South Korea were erroneously entered as being from North Korea or the Democratic People's Republic of Korea. This adjustment led to 318 articles. From these 318 articles, the countries of the co-authors, the affiliations of the North Korean authors, the journal titles, the number of articles over time, and the research fields of the articles were analyzed. The Hamhung Pharmaceutical University, Hamhung Chemical University, Hamhung Hydraulic Power University, Hamhung University of Chemical Industry, and Hamhung University of Chemical Technology were treated as a single institution (Hamhung University). From the 318 articles, those published without collaboration with researchers from other countries were selected as a subset. Forty-six articles were written by North Korean authors only. The same bibliometric parameters were analyzed, and the funding agencies were also analyzed.

Word cloud was built using tm and wordcloud packages of $\mathrm{R}$ available from: http://cran.nexr.com. Out of corpus, numeric, punctuations, stop words were removed. Capital characters were transformed into small characters. Following words were removed: results, using, can, based, properties, two, method, paper, also, elsevier, reserved, study, analysis, used, obtained, rights, different, show, new, investigated, well, conditions, data, incline, order, present, approach, degrees, one, respectively, experimental, found, solution, showed, number, three, korea, states, ltd, model, and investigated. After that, upper ranked 50 words from each corpus were included in word cloud with at least 5 times appearance. At first, word clouds of all 318 articles and 46 North Korean authors only articles were built. To find the recent trend, those of the 126 articles published through 2010 and the 182 articles from 2011 to 2016 were built.

Descriptive statistics was applied to this cross-sectional observational study of the literature.

\section{Results}

The major collaborating countries were China (197, 61.95\%), Germany (50, 15.7\%), Australia (10, 3.2\%), the United States $(5,1.6 \%)$, and Italy $(4,1.3 \%)$ (Fig. 1, Suppl. 1). Three articles were co-authored with South Korean researchers. The 318 articles corresponded to 48 affiliations in North Korea (Suppl. 2). Kim Il Sung University (161, 50.6\%), the Kim Chaek University of Technology $(44,13.8 \%)$, the Academy of Science (41, 12.9\%), and the University of Science $(26,8.2 \%)$ were highly productive institutions (Fig. 2). The top 3 journal titles in which articles were most frequently published were the International Journal of Systematic and Evolutionary Microbiology (8), Journal of High Energy Physics (7), and Linear Algebra and Its Applications (6) (Fig. 3, Suppl. 3). The number of articles published by North Korean authors dramatically increased to 52 in 2015 and 55 in 2016 (Fig. 4). The top 5 research fields were physics $(66,20.8 \%)$, mathematics $(61$, $19.2 \%)$, material sciences $(42,13.2 \%)$, chemistry $(31,9.8 \%)$, and engineering (29, 9.1\%) (Fig. 5, Suppl. 4). Citation data 


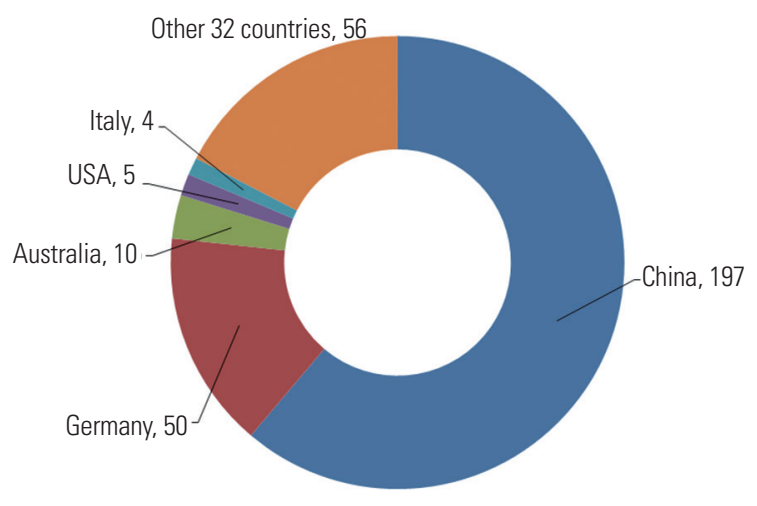

Fig. 1. Countries of the co-authors of articles by North Korean authors from the Web of Science Core Collection [cited on February 2, 2017].

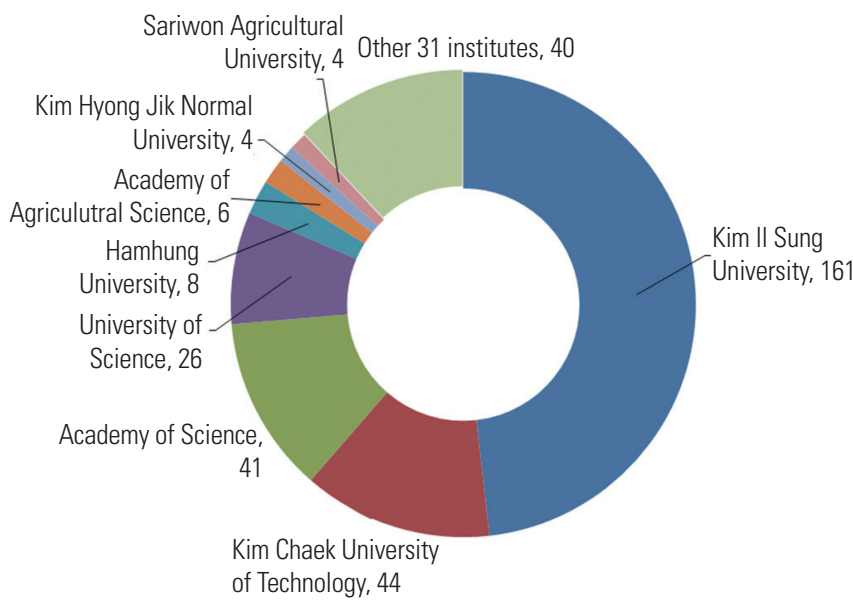

Fig. 2. Affiliations of the North Korean authors of the articles from the Web of Science Core Collection [cited on February 2, 2017].

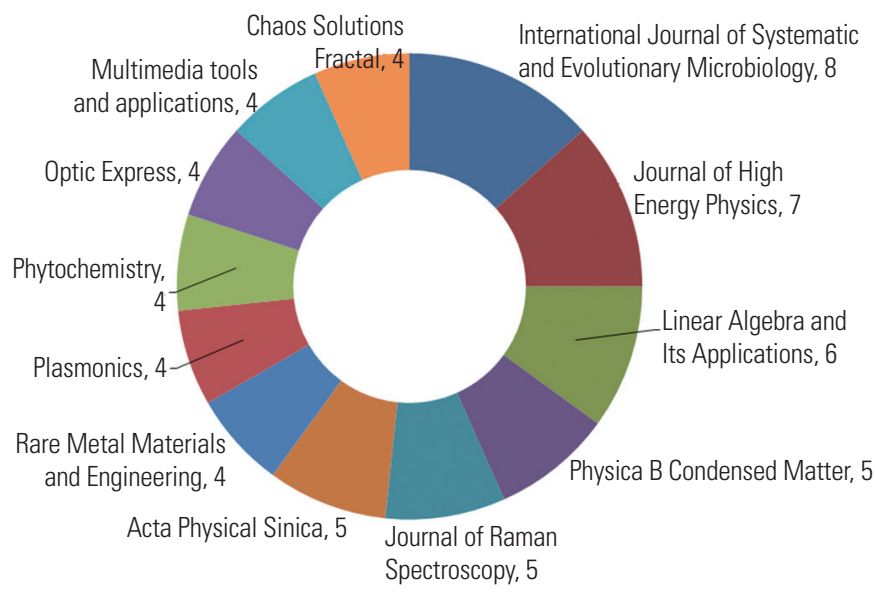

Fig. 3. Top 12 journal titles of articles by North Korean authors from the Web of Science Core Collection [cited on February 2, 2017].

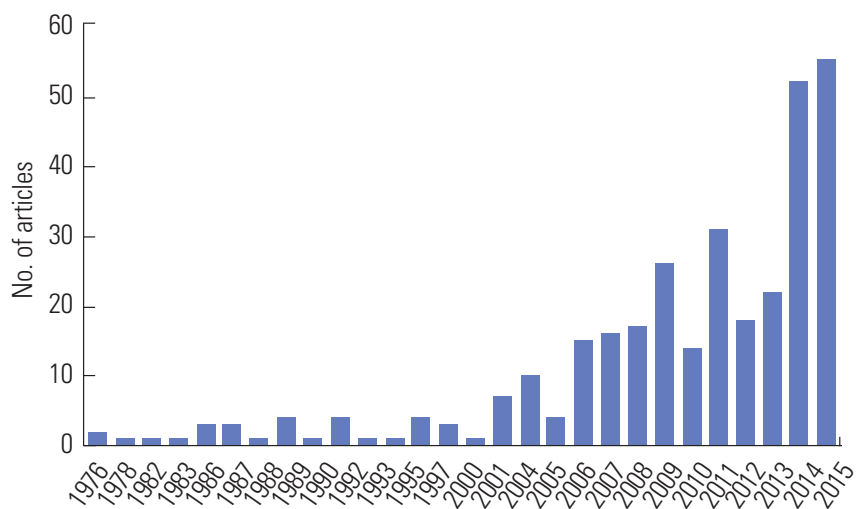

Year

Fig. 4. Number of articles by North Korean authors from the Web of Science Core Collection over time [cited on February 2, 2017].

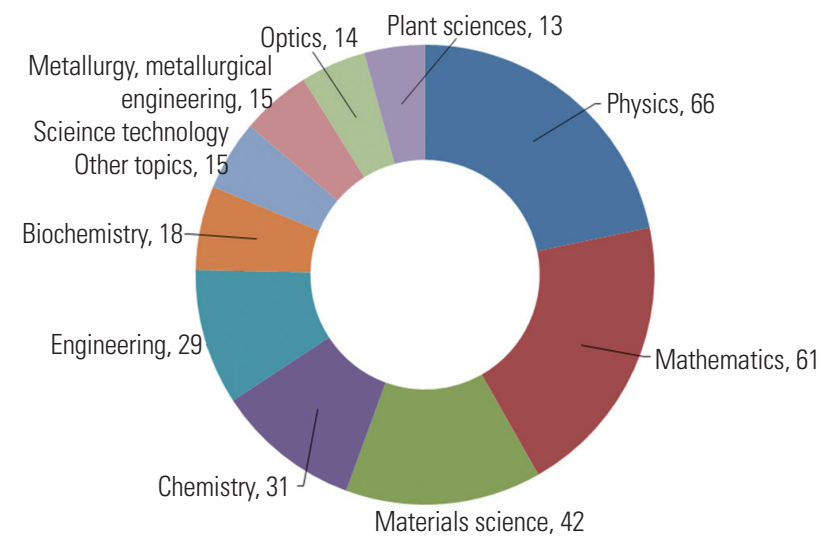

Fig. 5. Top 10 research fields of articles by North Korean authors from the Web of Science Core Collection [cited on February 2, 2017].

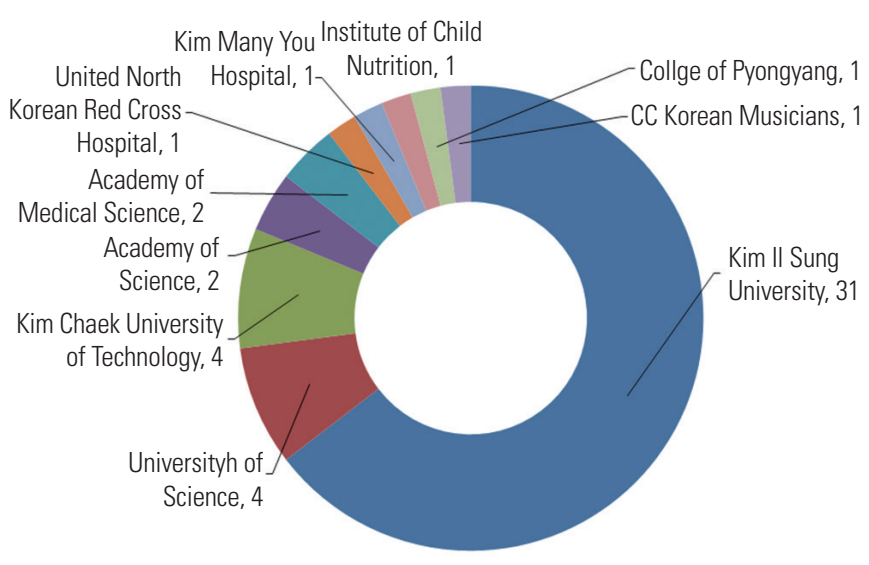

Fig. 6. Affiliation of the 46 articles with North Korean authors only from the Web of Science Core Collection [cited on February 2, 2017]. 


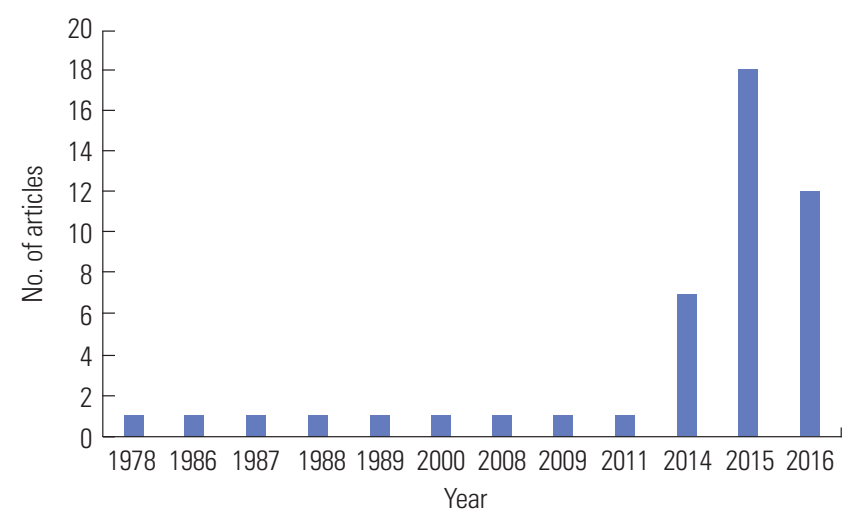

Fig. 7. Number of articles by North Korean authors only from the Web of Science Core Collection over time [cited on February 2, 2017].

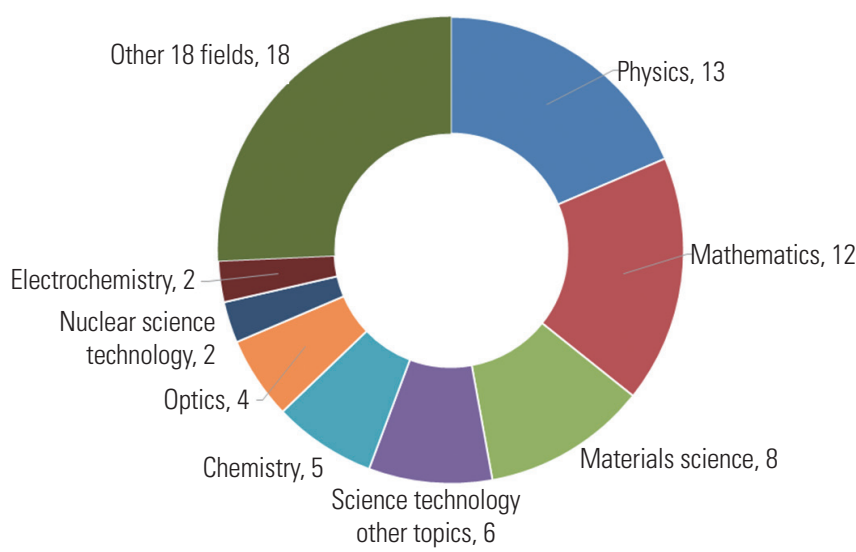

Fig. 8. Research fields of the 46 articles by North Korean authors only from the Web of Science Core Collection [cited on February 2, 2017].

and abstracts of 318 articles were available from Suppl. 5 .

Forty-six articles were published without co-authors from other countries. Of these articles, 32 (69.6\%) were from Kim Il Sung University, followed by the University of Science (4) and the Kim Chaek University of Technology (4) (Fig. 6). The journal titles are listed in Suppl. 6. The number of such articles recently increased to 18 in 2015 and 12 in 2016 (Fig. 7). The major research fields of articles by North Korean authors only were physics (13), mathematics (12), material sciences (8), science and technology other topics (6), and chemistry (5) (Fig. 8). Of those 46 articles, 9 were funded: 7 by the Ministry of Education of North Korea; 1 by the World Academy of Science for the Advancement of Science in Developing Countries (TWAS); and 1 by TWAS; the United Nations Educational, Scientific and Cultural Organization (UNESCO); and the Academy of Mathematics and Systems Science of the Chinese Academy of Sciences.

Word clouds are presented in Figs. 9 and 10. There were differences of corpus between all 318 articles and 46 North
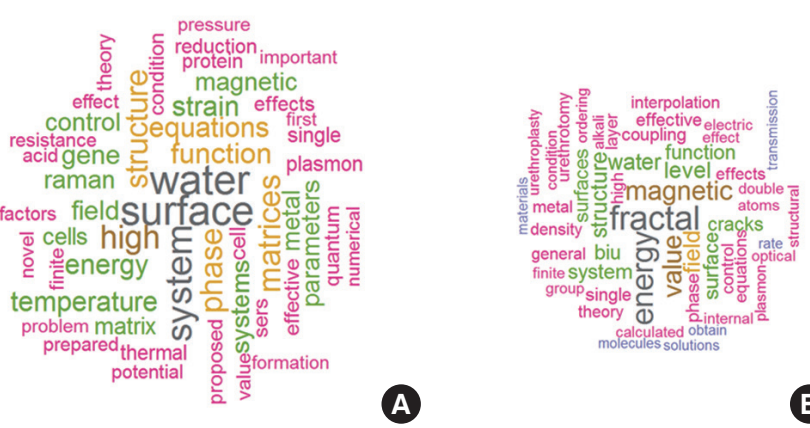

Fig. 9. Word clouds of (A) all 318 articles by authors from North Korea and (B) the 46 articles by North Korean authors only from the Web of Science Core Collection from 1988 to 2016.
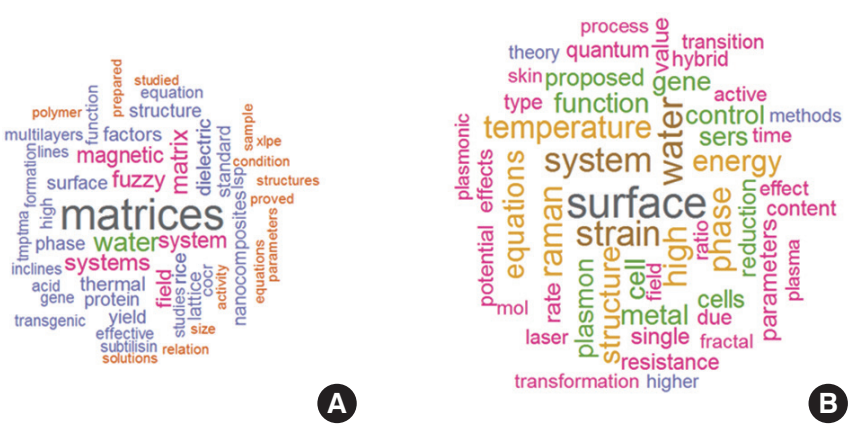

Fig. 10. Word clouds of $(A)$ the 126 articles published through 2010 and $(B)$ the 182 articles from 2011 to 2016 by authors in North Korea from the Web of Science Core Collection from 1988 to 2016.

Korean authors only articles: in the latter, the terms related with physics appear more frequently (Fig. 9). In the corpus from 126 articles published through 2010 and from articles from 2011 to 2016, the major terms were related with physics. the only difference was more number of agricultural terms in the former and more frequency of biology terms in the latter (Fig. 10). Raw data were available from Suppls. 7 to 10.

\section{Discussion}

The above results show that the articles by North Korean authors were mostly co-authored with researchers from other countries (85.5\%). Of the 318 articles, 46 (14.5\%) were by North Korean authors only. These are very small numbers given the population ( 25 million), gross domestic product (40 billion US dollars), and research activities of North Korea. We can infer the following conclusions: research results have been published mostly through journals in North Korea, cooperative or collaborative research with researchers in other countries has been very limited, and a few researchers in North Korea can access the internet since they presumably submit the 
manuscripts to international journals through the internet.

North Korean authors most frequently collaborated with Chinese researchers (Fig. 1). The next most common countries of collaboration were Germany and Australia. The importance of China as a collaborating partner is thought to originate from the fact that China is the geographic neighbor of North Korea and a fellow socialist country; therefore, China has invited North Korean researchers to their institutions. Kim Il Sung University, the Kim Chaek University of Technology, and the Academy of Science were the top-ranking institutions in North Korea in terms of articles published in Web of Science journals (Fig. 2). These 3 institutions published 246 (77.4\%) of the articles. It is believed that the North Korean government has implemented special strategies to promote research at these institutions. The journal list was compatible with the research fields; that is, journals from the fields of physics, mathematics, and material science were predominant (Fig. 3). Of the 9 articles in the field of biology, 8 were published in the International Journal of Systematic and Evolutionary Microbiology (Fig. 5). An increasing trend in article publication was evident, although the baseline was very low. The fact that more than 50 articles were published in 2015 and 2016 indicates that the North Korean government has begun to promote research and development and to recommend publication in international journals (Fig. 4). One article was published in the field of the arts, and 1 in the social sciences. It is believed that both arts/humanities and social sciences researchers have very rare chances to study abroad and produce collaborative works with researchers from other countries. The research and development strategy of the North Korean government has been focused on the physical sciences and engineering. There were 19 articles in the medical field and 16 in agriculture (Suppl. 1).

When the 46 articles published by North Korean authors only were analyzed, similar patterns were observed: the same top 3 institutions (Fig. 6), a surge of publication in 2015 and 2016 from a very small baseline (Fig. 7), and the same research fields (Fig. 8). Seven of the funded articles were funded by the Ministry of Education of the North Korean government; while 2 were supported by international funds such as TWAS and UNESCO, and 1 Chinese funding agency. The other 35 articles (76.1\%) had no funding. The proportion of funded articles was insufficient. It is believed that many researchers in North Korea can engage in research and development using their basic facilities and manpower. Within the 46 articles with North Korean authors only, one conspicuous researcher was Song-Jin Im, professor of physics, Kim Il Sung University. He published 6 of those 46 articles, and was only researcher to provide ORCID (http://orcid.org/0000-00016277-7200) information among the authors of those 46 arti- cles. According to his career summary on ORCID, he received a $\mathrm{PhD}$ from Kim Il Sung University in 2005, and moved to the Max Born Institute for Nonlinear Optics and Short Pulse Spectroscopy, Germany from 2008 to 2010. According to the physicist Professor Kihong Kim from Ajou University in South Korea, he was an excellent researcher in Germany and his research activity is comparable to that of top-level South Korean physicists. We can verify that although the North Korean government has restricted most North Koreans from accessing the internet outside of North Korea, it allows topnotch researchers at selected research institutions to access the internet of other countries. Although the Korean government recommended collaboration between North Korean and South Korean researchers from 1998 to 2007, only 3 collaborative research articles were found in the Web of Science. Collaborative work between North and South Korean researchers has remained limited due to political tensions.

A report on the bibliometric analysis of the articles from North Korea indexed in the Web of Science and Scopus from 1975 to 2012 has been published [1]. A total of 251 articles were analyzed. However, in the present study, the number of articles up to 2012 was. This discrepancy between the 2 studies may originate from differences in the included databases, as the previous report also included Scopus. There may have also been a difference in the selection criteria for institutions. We manually selected articles whose authors had affiliations with institutions in North Korea. Although we first found 401 hits, this number decreased to 318 after implementing this inclusion criterion. In the previous study, the most frequent collaborating countries were China, Germany, and Australia. The 3 most productive institutions were Kim Il Sung University, the Academy of Science, and the Kim Chaek University of Technology. The major research fields were physics, engineering, and chemistry. There was a remarkable increase of number of articles in 2012, from a very small baseline [1]. In contrast, in this study the major fields were found to be physics, mathematics, and materials science.

Word cloud shows the main terms in certain corpus. Comparison of word clouds between different groups of literatures was done. In all word clouds, terms related with physics were dominant. This trend has been strengthened in the articles by North Korean authors only (Figs. 9, 10).

This study had some limitations. First, to precisely characterize research trends in North Korea, the journals published in North Korea should be analyzed. Articles in the Emerging Sources Citation Index were not included. If the Emerging Sources Citation Index had been included, the number of articles from North Korea would have been greater than reported in this study. Content analysis with data mining is another topic to be explored in the analysis of research trends. 
In conclusion, our hypotheses were accepted, since the main research area was the physical sciences, including physics, mathematics, and materials science, and there was a surge in the number of articles in 2015 and 2016 from a very small baseline. Kim Il Sung University was the top-ranking institution, and has produced many articles in international journals. Additionally, top-notch researchers in North Korea are believed to use the internet outside of North Korea without difficulty during their research work.

\section{Conflict of Interest}

No potential conflict of interest relevant to this article was reported.

\section{Supplementary Material}

Supplementary files are available from: https://doi.org/10.7910/ DVN/VG9F2U

Suppl. 1. List of countries from 318 articles by North Korean authors from the Web of Science Core Collection [cited on February 2, 2017].

Suppl. 2. List of institutes from 318 articles by North Korean authors from the Web of Science Core Collection [cited on February 2, 2017].

Suppl. 3. List of journals from 318 articles by North Korean authors from the Web of Science Core Collection [cited on February 2, 2017].

Suppl. 4. List of research fields from 318 articles by North Korean authors from the Web of Science Core Collection [cited on February 2, 2017].

Suppl. 5. Citation data including title, authors, and abstract from 318 articles by North Korean authors from the Web of Science Core Collection [cited on February 2, 2017].

Suppl. 6. Citation data including title, authors, and abstract from 46 articles by North Korean authors only from the Web of Science Core Collection [cited on February 2, 2017].

Suppl. 7. Words and their frequencies from titles and abstracts of 318 articles by North Korean authors from the Web of Science Core Collection [cited on February 2, 2017].

Suppl. 8. Words and their frequencies from titles and abstracts of 46 articles by North Korean authors only from the Web of Science Core Collection [cited on February 2, 2017].

Suppl. 9. Words and their frequencies from titles and abstracts of 126 articles published through 2010 by North Korean authors from the Web of Science Core Collection [cited on February 2, 2017].

Suppl. 10. Words and their frequencies from titles and abstracts of 126 articles published 182 articles from 2011 to 2016 by North Korean authors from the Web of Science Core Collection [cited on February 2, 2017].

\section{Reference}

1. Shelton RD, Lewison G. Scientific collaboration as a window and a door into North Korea. Scientometrics 2013;97: 3-11. http://dx.doi.org/10.1007/s11192-012-0946-8 